\title{
Psychological Resistance for Insulin Therapy Among Patients with type 2 Diabetes Mellitus: a Cross Sectional Survey.
}

\author{
Thillainathan S, Pratheep N, Marasinghe IUK, Karunayokiny K, Gunasinghe KP, Priyangika DK³, Premawansa G.
}

\begin{abstract}
Introduction: Psychological insulin resistance can result in delays in treatment initiation. The aim of our study is to find the prevalence of psychological insulin resistance among patients at a tertiary hospital in Sri Lanka.

Materials and methods: A prospective cross sectional survey was conducted at General Medical clinic, Colombo North Teaching Hospital, using a pre-tested, intervieweradministered 25 question survey adapted from Insulin Treatment Appraisal Scale (ITAS) among a consequent sample of 358 patients.
\end{abstract}

Results: Majority (52.2\%) of patients considered commencement of insulin therapy as personal failure $(P=0.0001)$. Gender had a significant impact on the fear for needle with females having a higher negative score (3.39) compared to males (3.18) $(P=0.040)$. Overall negative appraisal for insulin was similar in both genders. $49.1 \%$ feared that once insulin is started it is needed permanently while $0.8 \%$ opposed it. Statistically significant difference was not found between the total mean score for negative items among patients on OHDs (3.19) and patients on insulin (3.10) ( $P=0.133)$. Perception of social stigmata and perception of injection conveyed higher negative appraisal. Educational level and duration of diabetes did not have significant influence on insulin perception.

Conclusion: Health care workers need to probe into the perceptions that worry the patients and focus on groups with more negative appraisal towards insulin.

\section{Introduction}

Globally, the prevalence of chronic, non-communicable diseases is increasing at an alarming rate. Consequently, diabetes is rapidly emerging as a global health care problem that is threatening to reach pandemic levels by 2030; the number of people with diabetes worldwide is projected to increase from 171 million in 2000 to 366 million by 2030. [1] Recent studies have shown that diabetes is not restricted to the industrialized countries. Actually it is the population in developing countries, and the minority of disadvantaged communities in the industrialized countries who now face the greatest risk. [2]

1. General Medical Unit, North Colombo Teaching hospital, Ragama, Colombo.
The prevalence of diabetes mellitus shows an increasing trend in Sri Lanka. In 1993, the prevalence of diabetes in rural Sri Lankan community was 2.5\% [3] and this has rapidly escalated to $13-14 \%$ representing around $500 \%$ increase in prevalence rates over a 10 year period. [4] The pathogenesis of type 2 diabetes mellitus is characterized by insulin resistance followed by gradual decline in beta cell function. Actually at the time of diagnosis the pancreatic beta cell function of type 2 diabetes patients is found to be only 50\%. [5] Further decreases in beta cell function occurs as the disease progresses and at some point most type 2 diabetes patients will need insulin treatment. UKPDS trial demonstrated that $53 \%$ of patients initially treated with a sulphonylurea eventually needed insulin therapy within 6 years of follow up to maintain adequate glycaemic control. [6]

A study conducted by Okazaki et al reveals that when a diabetic patient is offered insulin therapy, more than one quarter of them refuses it initially. [7] Clinicians tend to relate patients refusal for insulin towards needle phobia but according to the literature, psychological insulin resistance may arise due to various factors such as patients' feelings of personal failure to effectively self-manage their diabetes, feeling taking insulin means their disease has become worse, or as it makes life less flexible. This psychological insulin resistance can result in delays in treatment initiation.

Initiating insulin therapy without targeting these issues would lead to poor patient compliance, sub optimal glycaemic control or even to fall out of the whole treatment regime. Local Sri Lankan studies are not available in this field. Cultural beliefs may play a role in an individual's perception regarding diseases and treatment. The aim of our study was to find the perception regarding insulin therapy among patients with type 2 diabetes mellitus at a tertiary hospital in Sri Lanka. Also the perception towards insulin among insulin naive and insulin using groups was compared and the reasons for the perception towards insulin therapy were analysed.

\section{Materials and methods}

A prospective cross sectional survey was conducted at General Medical clinic, Colombo North Teaching Hospital in September 2014, using a pre-tested, intervieweradministered 25 question survey adapted from Insulin 
Treatment Appraisal Scale (ITAS). [8] Patients attending the medical clinic during the study period who have been diagnosed as having type 2 diabetes mellitus and receiving oral hypoglycaemic drugs or injectable insulin or both for more than three months duration from the clinic were included for the study. 358 patients were selected by systematic sampling method. Type 1 diabetes mellitus patients were excluded from the study. Data was computerized and analysed according to the objectives using simple descriptive statistics.

\section{Results}

The study was conducted among 358 patients with a mean age of 60.1 years $(S D=12.21)$. Out of the total study sample $233(65.1 \%)$ were male.Two hundred and seventy two $(76 \%)$ of them were on oral hypoglycaemic drugs (OHDs) whereas 86 (24\%) were on insulin therapy. Considering the educational level of the patients $41.1 \%$ of patients had an educational level of less than grade 5 while $37.2 \%$ had studied up to ordinary standard or above. Hundred and eighty six (52\%) had a low income of less than Rs 10000 per month whereas only 5 (1.4\%) had a monthly income of Rs 30000 or above.

Perception of disease progression Majority (52.2\%) of patients considered commencement of insulin therapy as personal failure while $1.4 \%$ opposed it. They perceived that prescribing of insulin means they have failed to properly care for their diabetes with tablets and diet and this appraisal was statistically significant $(P=0.0001)$

Perception of injection About $41.9 \%$ of patients agreed or strongly agreed that insulin injection is painful whereas only $7.3 \%$ disagreed. Majority (32.1\%) accepted that they feared the needle while $15.1 \%$ opposed it. Interestingly, may be as expected, gender had a significant impact on the fear for needle. Females had a higher negative score for the fear of needle (3.39) compared to males (3.18) which was statistically significant $(\mathrm{P}=0.040)$.

Perception of lifestyle modification A high proportion of patients ( $38 \%$ vs. $6.4 \%$ ) feared that starting insulin therapy would make them more dependent on doctors and it makes lifestyle more inflexible. In contrast to it, for the statement "I have to give up activities I enjoy", majority $(13.4 \%$ vs. $9.6 \%)$ disagreed. However this finding was not statistically significant.

Perception of insulin related adverse effects Majority of patients (36.9\% vs. $3.1 \%)$ feared that insulin usage may increase the risk of hypoglycemia. Similarly insulin was also perceived by a higher proportion of patients (36.4\% vs. $7.2 \%$ ) that it would deteriorate the health.

Perception of social stigma In contrast to the general belief, only a handful of patients $(2.8 \%)$ viewed insulin injection as embarrassing while $22.7 \%$ denied it. On the other hand a high proportion of patients ( $41.9 \%$ vs. 7.3\%) were anxious that starting insulin might lead their family to get more concerned about them.

Perception of therapy About $49.1 \%$ feared that once insulin is started it is needed permanently while $0.8 \%$ opposed it. $(p=0.001)$. Similarly a significant proportion of patients $(31.3 \%)$ preferred to delay insulin till it is absolutely necessary while $7.8 \%$ opposed it $(p=0.001)$.

Perception of diabetes control Generally patients held a positive attitude towards insulin therapy and disease control. About 27.3\% expressed that insulin helps to prevent complications of diabetes, $70.5 \%$ were neutral while only $2.2 \%$ refused it. Similarly, the statement "insulin helps to maintain good control of blood glucose" was accepted by $28 \%$ while opposed by $2.8 \%$. Nevertheless "insulin improves energy level" was denied by $13.1 \%$ while $7.6 \%$ accepted it.

Overall, perception of social stigmata and perception of injection conveyed higher negative appraisal. Interestingly educational level and duration of diabetes did not have significant influence on insulin perception and the overall negative appraisal for insulin was found to be similar in both genders. Finally, in contrast to the general belief, statistically significant difference was not found between the total mean score for negative items among patients on oral hypoglycaemic drugs (3.19) and patients on insulin (3.10) ( $P=0.133)$.

\section{Discussion}

When oral medications fail, insulin has a definitive benefit in type 2 diabetes mellitus patients. Nevertheless some reluctance is found among both patients and healthcare professionals to commence insulin therapy. This reluctance has been termed "psychological insulin resistance". Patients with type 2 diabetes are often reluctant to begin insulin for various reasons and tend to delay the start of insulin therapy for quite lengthy periods of time. Actually patients may not appreciate any reassurances or additional information unless their personal beliefs and attitudes about insulin are recognized and discussed. So it is of utmost important that these negative attitudes should be identified and properly addressed prior to the commencement of insulin.

In our study, the attitudes expressed by the patients gave some insight into the reasoning behind the reluctance to begin insulin treatment. Most accepted that insulin prevents complications and helps to maintain good control of blood glucose levels. However, these benefits were often obscured by negative attitudes regarding insulin. Barriers to the insulin therapy included fear of disease progression, needle anxiety and the possible 
dangers of taking it. They also indicated that insulin holds a negative symbolic mystique for them, representing failed treatment and advancing illness. Not surprisingly, when patients are convinced that insulin will worsen their health, they may be very resistant to initiate insulin therapy.

Majority (52.2\%) of our patients considered commencement of insulin therapy as personal failure. Similar results were found in few other studies. A study by Hunt et al. revealed nearly a third of patients $(32 \%$, $14 / 44$ ) associated insulin therapy with personal failure [9] while in few other studies, as many as $50 \%$ of patients perceived it as a personal failure and a well-deserved punishment for one's own negligence in diabetes selfcare. $[10,11]$ Patients with diabetes mellitus tend to perceive being prescribed insulin means the disease has progressed into a serious phase. Injecting insulin carries a great symbolic load indicating that the condition has become grave, that past treatment efforts have failed, and that the patient has not taken proper care of their disease.

In our study, a high proportion of patients (38\% vs. $6.4 \%$ ) feared that starting insulin therapy would make them more dependent on doctors and it makes lifestyle more inflexible. A study by Polonsky et al revealed similar results where $50.6 \%$ of patients believed that insulin therapy would restrict their lives. [10] Insulin is often associated with a perceived loss of control over one's life. Another important factor identified was concerns about injection related anxiety. Among our study population, $41.9 \%$ of patients agreed or strongly agreed that insulin injection is painful whereas only $7.3 \%$ disagreed. This was in agreement with a study by Skovlund et al where approximately $50 \%$ of patients reported fearful of injections. [11] Nevertheless patients' attitude towards insulin avoidance is not just a simple fear of needles but extends far beyond to include deeply held beliefs about insulin.

In our present study, statistically significant difference was not found between the total mean score for negative items among patients on OHDs (3.19) and patients on insulin (3.10) $(\mathrm{P}=0.133)$. These results were in contrast to that reported by Snoek et al among Caucasians type 2 diabetes patients, where insulin naïve patients reported significantly higher scores for the negative items compared to insulin treated. [8] Further studies are warranted to identify the extent of attitudinal changes experienced following initiation of insulin in diabetes patients.

In conclusion, psychological resistance to insulin can be prevented by identifying patients' personal obstacles to insulin treatment and addressing them accordingly. Health care workers need to probe into the perceptions that worry the patients and focus on groups with more negative appraisal towards insulin and help them to see insulin as a potential friend than a foe.

\section{References}

1. Wild S, Roglic G, Green A, et al. Global prevalence of diabetes: estimates for the year 2000 and projections for 2030. Diabetes Care 2004; 27(5):1047-53.

2. King $\mathrm{H}$, Rewers $M$. Diabetes in adults is now a Third World problem. The WHO Ad Hoc Diabetes Reporting Group. Bull World Health Organ 1991; 69(6):643-8.

3. Illangasekera U, Nugegoda DB, Perera LS. Prevalence of diabetes mellitus and impaired glucose tolerance in a rural Sri Lankan community. Ceylon Medical Journal1993; 38(3):123-6.

4. Wijewardene KMM, Mendis S, Fernando DS, et al. Prevalence of hypertension, diabetes and obesity: baseline findings of a population based survey in four provinces in Sri Lanka. The Ceylon Medical Journal 2005 Jun; 50(2): 62-70.

5. UK Prospective Diabetes Study Group. UK prospective diabetes study 16. Overview of 6 years' therapy of type II diabetes: A progressive disease. Diabetes 1995; 44:1249-58.

6. Wright A, Burden AC, Paisey RB, et al, for the UK Prospective Diabetes Study Group. Sulfonylurea inadequacy: Efficacy of addition of insulin over 6 years in patients with type 2 diabetes in the UK Prospective Diabetes Study (UKPDS 57) [published correction appears in Diabetes Care 2002; 25:1268] Diabetes Care 2002;25:330-36.

7. Okazaki K, Goto M, Yamamoto T, Tsujii S, Ishii H. Barriers and facilitators in relation to starting insulin therapy in type 2 diabetes. Diabetes 1999; 48 (Suppl.1): A 319.

8. Snoek FJ, Skovlund SE, Pouwer F. Development and validation of the insulin treatment appraisal scale (ITAS) in patients with type 2 diabetes. Health and Quality of Life Outcomes 2007; 5: 69-74.

9. Hunt LM, Valenzuela MA, Pugh JA. NIDDM patients' fears and hopes about insulin therapy: the basis of patient reluctance. Diabetes Care 1997; 20:292-98.

10. Polonsky WH, Fisher L, Dowe S, Edelman S. Why do patients resist insulin therapy? Diabetes 2003; 52: A417.

11. Skovlund SE, van der Ven N, Pouwer F, Snoek F. Appraisal of insulin treatment in type 2 diabetes patients with and without previous experience of insulin therapy. Diabetes 2003; 52: A419. 